\title{
Rôle de l'endettement extérieur dans le financement du développement économique : cas des pays de la région MENA
}

\author{
Tarek BEN ALI ${ }^{1}$, Ahmed Zidi ${ }^{2}$ \\ Institut Supérieur d'Administration des Entreprises, Université de Gafsa - Tunisie \\ 1 tarekbenal@yahoo.fr \\ 2 ahmed_zidi@yahoo.fr
}

\begin{abstract}
En considérant les économies de la région MENA, l'objet de cette recherche est d'étudier le rôle de l'endettement extérieur dans le financement du développement. II s'agit en particulier d'étudier la relation entre la dette extérieure et la croissance économique. En fait, les changements politico-économiques que connaît le Sud de la Méditerranée combinés aux urgences dictées par la crise de la dette souveraine en Europe fait qu'il est pertinent de s'interroger sur la réponse à apporter aux défis économiques que doit relever la région MENA dans son ensemble. Au premier rang de ces défis figure la problématique de l'endettement extérieur et son rôle dans le financement de développement économique.

L'étude s'est inspirée du modèle de Patillo et al. (2002) et elle utilise la technique de panel. Ainsi et en combinant des ratios d'endettement et les principaux déterminants de la croissance économique, nous avons vérifié l'existe d'un seuil optimal au-delà duquel l'endettement extérieur ralentit la croissance économique (l'existence d'une courbe de Laffer de la dette). Pour la région MENA, la dette extérieure en soit n'est pas un frein au développement lorsqu'elle est contenue dans des limites raisonnables; au contraire, elle peut aider ces pays à consolider leur croissance. Par ailleurs, une augmentation du service de la dette a un impact négatif sur la croissance économique et le canal de transmission de cet impact passe par la qualité de l'investissement et par le fardeau de la dette. Les résultats obtenus montrent également qu'il existe une relation non linéaire entre la dette et la croissance, c'est-à-dire qu'il y a un seuil critique qui rendrait la croissance négative. Ce seuil, qui correspond à l'impact marginal de la dette sur la croissance s'établit à un niveau de $60 \%$ du PIB.
\end{abstract}

\section{Indexing terms/Keywords}

Endettement extérieur, croissance économique, pays de la région MENA, données de Panel

\section{Council for Innovative Research}

Peer Review Research Publishing System

Journal: International Journal of Management \& Information Technology

\author{
Vol. 7, No. 3 \\ editor@cirworld.com \\ www.cirworld.com, member.cirworld.com
}




\section{INTRODUCTION}

Les problèmes socioéconomiques que connaissent actuellement les pays arabes suite à leur révolution contre la dictature conjugués à la crise de la dette souveraine en Europe ont ravivé le débat sur le rapport entre l'emprunt extérieur et la croissance économique. En fait, L'endettement extérieur a été toujours considéré comme une conséquence naturelle des activités économiques. II vient du fait que certains pays ou institutions ont des excédants financiers et d'autres ont des besoins de financement. L'endettement permet donc à un pays d'investir des capitaux au-delà de ses propres disponibilités financières en empruntant des excédants de capitaux (Klein, 1994). La dette ainsi créée est supposée, toutes choses égales par ailleurs, générer la croissance et favoriser le développement. Cependant, la crise de la dette des années 1980 a remis en cause l'hypothèse selon laquelle le recours aux emprunts internationaux permet d'accroître les investissements et de promouvoir le développement.

Concernant les pays développés, la récession actuelle plus ou moins grande qui règne dans ces pays depuis l'éclatement de la crise de l'endettement européen, laisse à croire qu'il existe un rapport négatif entre l'importance de l'endettement et la croissance économique. Cette corrélation a été confirmée par plusieurs études citées à maintes reprises et qui a été présentée en 2010 par Carmen Reinhart et Kenneth Rogoff. Ces auteurs, ont analysé des données collectées sur de très longues périodes et relatives à la croissance, l'inflation et le taux d'endettement de 20 pays industrialisés et 24 pays en développement (PED). Tant pour les pays avancés que pour les économies émergentes, ils ont démontré qu'avec un taux d'endettement supérieur à $90 \%$ du produit intérieur brut (PIB), la relation entre dette publique et croissance économique est faible, mais au-delà de cette limite la croissance est largement affaiblie. Cependant, pour les pays en développement le seuil de dette publique détenue par l'extérieur est plus faible, avec une chute de deux points de la croissance quand le ratio dette publique détenue par l'extérieur/PIB dépasse $60 \%$. La croissance devient négative quand ce ratio excède 90 $\%$.

Dans ce même contexte et en cherchant s'il existe une limite maximale à l'endettement public au-delà duquel l'État ferait faillite, certain nombre d'arguments théoriques (Krugman 1988, Sachs 1989, Pattillo et al. 2002, Clements et al. 2003) ont montré qu'une hausse du ratio de dette publique pourrait conduire à une réduction de la croissance à cause d'un effet d'éviction des investissements productifs et de la relative inefficacité de l'État dans l'utilisation des ressources. D'autres arguments sont souvent évoqués et se rapportent aux effets plus immédiats de l'augmentation de l'endettement de l'Etat. II s'agit surtout du problème de soutenabilité budgétaire qui pourrait conduire le gouvernement à se mettre en défaut de paiement ou à imposer une « taxe inflationniste » coûteuse impliquant un ralentissement de la croissance.

Au regard de ce qui précède, l'objet de cette étude consiste à évaluer l'impact de la dette extérieure sur le développement économique dans la région MENA. Pour ce faire, nous allons vérifier, premièrement, les effets de l'endettement extérieur à travers l'effet d'éviction du service de la dette et l'existence, ou non, d'un impact non linéaire de la dette sur l'investissement et la croissance dans les économies de cette région (relation entre la courbe de Laffer de la dette et la croissance). Ensuite, nous chercherons à savoir comment se manifeste cet effet de la dette sur ces économies (les canaux de transmission).

Le reste du papier sera organisé comme suit. La deuxième section sera consacrée à une revue de la littérature sur les enseignements théoriques relative à la relation entre l'endettement extérieur et la croissance économique. La troisième section présente le modèle retenu ainsi que les résultats des différents tests économétriques. La quatrième et dernière section conclut le papier.

\section{DETTE PUBLIQUE ET CROISSANCE ECONOMIQUE : LES ENSEIGNEMENT THEORIQUES}

Selon la théorie de surendettement, un endettement public, contenu dans des limites raisonnables, peut aider les pays concernés à consolider leur croissance. En effet, quand ces pays emploient les capitaux empruntés pour financer des investissements productifs, leur croissance devrait s'accélérer tout en leur permettant de rembourser à l'échéance les dettes contractées.

Cependant, lorsqu'il y a une probabilité future que la dette externe dépasse les capacités de remboursement du pays, les coûts prévus du service de la dette découragent davantage l'investissement domestique et étranger et défavorisent la croissance économique (Corden (1988), Krugman (1988), Sachs (1989), Pattillo et al. (2002)). Ainsi, la charge élevée du service de la dette accroît les taxes futures prévues sur le secteur privé ce qui réduit par conséquent l'investissement privé. Les ressources, qui au lieu d'être canalisées pour l'investissement, seront épongées par le fardeau du service de la dette. Par ailleurs, en changeant la qualité de l'investissement, le surendettement peut détériorer la performance économique et l'incertitude du remboursement du service de la dette constitue une difficulté pour poursuivre la réforme économique (Clements et al. 2003). 
Toutefois, la pertinence de la théorie de surendettement a été relativisée par certains auteurs qui voient que l'accumulation de la dette n'est pas la cause mais la conséquence d'une faible croissance (Bulow et Rogoff (1990)), ou que la réduction de la dette ne serait pas suffisante pour rétablir l'investissement et la croissance (Easterly (2002), Asiedu (2003)). Dans ce même contexte, d'autres auteurs considèrent que les problèmes des pays en développement en matière d'endettement se rapportent plutôt à la liquidité (liés au service de la dette) et non aux incitations liées à l'encours de dette (Cohen (1993), Claessens et Diwan (1989)).

II semble donc que l'impact de la dette extérieure sur la croissance n'a pas fait un consensus général entre les économistes. Pour déceler davantage la nature de cet impact, nous allons consacrer la section suivante aux différentes interprétations théoriques de la dette extérieure.

\subsection{La conception keynésienne de l'endettement}

L'approche keynésienne de la relation dette publique-croissance économique considère que l'impact des déficits publics autrement dit de l'augmentation des dettes publiques - sur la croissance est jugé positif. Ainsi et dans le cas d'une sousactivité, une augmentation des dépenses entièrement financée par les impôts, donc implicitement sans impact sur la dette publique, induit une hausse équivalente du PIB («Balanced Budget Multiplier»). Selon l'approche keynésienne, une diminution des prélèvements fiscaux financée par un endettement de l'Etat stimule la consommation de court terme. En effet, une diminution de la charge d'imposition (non accompagnée par une réduction des dépenses publiques actuelles ou futures), augmente le revenu disponible des ménages. Puisque la consommation, dans cette perspective keynésienne, est une fonction proportionnelle de ce revenu, alors l'effet de l'accroissement de ce dernier sur la consommation est positif. L'augmentation des dépenses de consommation va induire un accroissement de la demande agrégée, qui va se traduire par une augmentation équivalente de l'offre à l'équilibre du marché des biens et des services. In fine et toute chose étant égale par ailleurs, le déficit budgétaire financé par endettement public, implique un accroissement de la production et des revenus.

Cependant, l'approche keynésienne de l'endettement ne peut pas être valable à moyen et long terme. En effet, cette approche caractérisée par l'hypothèse de la rigidité des prix et des salaires ainsi que celle de la myopie temporaire des agents économiques ne peut fonctionner que dans le cadre d'un raisonnement de courte période. A long terme, les effets d'un déficit budgétaire menaçant la soutenabilité des finances publiques sont indéniablement négatifs. Ceux-ci incluent notamment une hausse des taux d'intérêt à long terme, en raison d'un accroissement de l'offre de titres publics placés sur le marché et d'une augmentation des primes de risque. De plus, l'accroissement des charges d'intérêts résultant de l'aggravation du déficit budgétaire réduit les dépenses publiques productives et augmente la pression fiscale et parafiscale. Autrement dit, l'endettement public se traduit, à long terme, par une réduction de l'épargne qui induit une contraction de l'investissement. Ceci affecte à son tour négativement l'offre et par voie de conséquence la production et les revenus.

Dans une logique de moyen et long terme, le canal de transmission de la dette sur la croissance se manifeste essentiellement par une diminution de l'investissement domestique et une contraction de l'activité du marché des capitaux. Ceci réduit la production et les revenus à cause d'une rareté des capitaux disponibles dans l'économie. En conséquence, la productivité marginale du capital devient plus élevée provoquant, ainsi, une augmentation des taux d'intérêt. Conjointement à cet accroissement de la rémunération du capital, la productivité du travail diminue, réduisant en conséquence le taux de salaire réel dans l'économie et les revenus du travail. Par ailleurs, la diminution de l'investissement net à l'étranger implique, d'une part, la réduction des revenus de capitaux des résidents placés à l'étranger et, d'autre part, la diminution des exportations nettes et donc une augmentation du déficit courant. II en résulte un déficit du compte courant ce qui induit une appréciation du taux de change et une détérioration de la compétitivité de l'économie nationale.

\subsection{La conception classique de l'endettement}

Par opposition à l'approche keynésienne, la théorie de l'équivalence ricardienne avancée par Robert Barro (1974) soutient, néanmoins, la thèse d'un effet neutre de l'endettement public sur la croissance économique. Selon cette approche, en finançant l'augmentation des dépenses publiques par le déficit, on peut s'attendre à ce que les impôts soient nécessairement augmentés pour servir ou rembourser la dette. En conséquence, les consommateurs et les entreprises augmentent leur taux d'épargne dans le but de disposer de moyens suffisants pour compenser les futures charges fiscales. Par ailleurs, des consommateurs rationnels perçoivent tout accroissement du déficit budgétaire courant, du à une réduction de leur charge d'imposition financée par endettement public, comme étant un accroissement de leur charge fiscale future, toute chose étant égale par ailleurs. En tenant compte de cette charge fiscale additionnelle future, les ménages ont tendance à maintenir leur comportement de consommation inchangé et préfèrent épargner la totalité de leur économie d'impôt pour faire face aux prélèvements fiscaux futurs engendrés par le remboursement de la dette. Donc, la 
diminution de l'épargne publique est intégralement compensée par une augmentation de l'épargne privée ce qui laisse inchangée l'épargne nationale.

En traitant la thèse de la proposition de l'équivalence ricadienne, les économistes se réfèrent souvent aux idées fondamentales de la contrainte budgétaire intertemporelle du gouvernement et de l'hypothèse du revenu permanent (Elmendorf et Mankiw (1998) et Ricciuti (2003)). Pour la contrainte budgétaire inter-temporelle du gouvernement, on suppose que pour des dépenses gouvernementales inchangées un niveau faible d'imposition courant signifie une augmentation plus élevée des impôts futurs. Autrement dit, les ménages perçoivent que toute réduction courante de leur charge d'imposition implique un report partiel de cette charge dans le futur.

Quant à l'hypothèse du revenu permanent, elle prévoit que les ménages déterminent leur niveau de consommation non pas sur la base de leur revenu courant mais plutôt à partir de leur revenu permanent. Ce dernier est fonction de la valeur actualisée de tous les revenus courants nets d'impôt. Puisque une diminution d'impôt qui sera financée par endettement n'affecte pas la valeur actualisée de la charge fiscale des ménages, elle n'aura pas d'incidence ni sur leur revenu permanent ni sur leur consommation courante.

\section{METHODOLOGIE D'ESTIMATION ET INTERPRETATIONS DES RESULTATS}

L'impact de la dette extérieure sur la croissance économique a fait l'objet de plusieurs recherches économétriques et la plupart des résultats obtenus confirment globalement la théorie de surendettement, en démontrant un impact négatif de la dette sur la croissance ( Agénor et Montiel (1999), Hansen (2001), Dijkstra et Hermes (2001), Clements et al. (2003), Idlemouden et Raffinot (2005), Swapan et al. (2007)). Un tel effet négatif se manifeste souvent à travers trois principaux canaux à savoir l'effet direct du poids élevé de la dette, l'effet d'éviction du service de la dette et l'effet de désincitation de l'encours de la dette. De ce point de vue, il est nécessaire d'alléger la dette extérieure des pays en développement (Deshpande (1997), Patillo et al. (2002), Clements et al. (2003), Cordella et al. (2005), Sen et al. (2007))). L'efficacité de sa réduction a été très souvent infirmée sur le plan empirique, en particulier lorsqu'elle a été mesurée par ses effets directs sur la croissance et pour les pays à faible revenu (Arslanalp et Henry $(2004,2005,2006)$, Depetris et Kraay (2005), Presbitero (2009) et Johansson (2010)).

D'autres travaux (Pattillo et al. (2004), Maghyereh et al. (2002), Kumar et Woo (2010), Reinhart et Rogoff (2010)) insistent sur l'influence positive de l'endettement sur la croissance en mettant en évidence une relation sous forme de $U$ renversé entre ces deux variables. Selon cette forme incurvée, la dette a un impact positif sur la croissance jusqu'à un certain niveau ; au-delà de ce niveau ou seuil, l'effet de la dette devient négatif.

\subsection{Spécification du modèle économétrique}

Pour la présente étude, le modèle utilisé par Patillo et al. En 2002 a servi de cadre de référence. En combinant des ratios d'endettement et les principaux déterminants de la croissance, ces auteurs ont abouti à des résultats satisfaisants. Notre objectif consiste, donc, à étudier l'impact de la dette extérieure sur la croissance économique et à vérifier s'il existe un seuil optimal au-delà duquel l'endettement extérieur ralentit la croissance économique (l'existence d'une éventuelle courbe de Laffer de la dette).

L'équation du modèle de croissance, en tenant compte de la disponibilité des données et des caractéristiques des économies de la région MENA se présente comme suit :

$$
\begin{aligned}
& \text { TCPIBH }_{i, t}=\alpha_{i}+a_{1} \text { TCPIBHR }_{i, t}+a_{2} \text { TCD }_{i, t}+a_{3} \text { TINV }_{i, t}+a_{4} \text { DCH }_{i, t}+a_{5} \text { VTE }_{i, t}+a_{5} \mathrm{OUV}_{i, t}+ \\
& a_{6} \text { SDEXP }_{i, t}+a_{7} \text { DETPIB }_{i, t}+a_{8} \text { DETEXP }_{i, t}+a_{9} \text { TINF }_{i, t}+\varepsilon_{i, t}
\end{aligned}
$$

Ainsi, la croissance économique (variable endogène) sera approximé par le taux de croissance du PIB réel par habitant. Comme facteurs explicatifs, nous avons retenu le revenu par habitant décalé, des taux d'investissement, de scolarisation dans le secondaire, de la croissance démographique (tous en logarithme), de la différence des politiques suivies (ouverture), ainsi que des chocs extérieurs (termes de l'échange). Pour cerner les effets de la dette, nous avons ajouté au modèle de croissance standard trois variables communément utilisées : la valeur nominale de l'encours de la dette publique extérieure, exprimée en pourcentage du PIB et en pourcentage des exportations de biens et de services, et le service de la dette en pourcentage des exportations.

Selon la spécification précédente, les variables d'intérêt portent sur le stock de la dette et son service. Ces variables nous permettent de tester les deux principales hypothèses de ce papier. La première hypothèse revient à examiner si le paiement d'un service de dette très élevé, en évinçant les ressources destinées à l'investissement, représente un frein à la croissance. Quand à la seconde hypothèse, elle s'intéresse à l'effet du paiement futur de la dette décrit par son encours. Pour vérifier l'existence ou non d'une relation non linéaire entre la dette extérieure et la croissance économique et déterminer le seuil d'endettement optimal, nous allons suivre deux spécifications (linéaire et quadratique). Concernant 
la première, elle suppose une relation linéaire, et chaque indicateur de la dette est introduit en sa forme normale. Pour la seconde spécification, elle intègre successivement les variable dette extérieure en pourcentage du PIB et en pourcentage des exportations des biens et services levées au carré.

\subsection{Présentation de l'échantillon et définition des variables explicatives}

L'échantillon retenu se compose de 8 PED apparentant à la région MENA dont six pays faisant partie des économies du Moyen-Orient (Jordanie, Liban, République Arabe syrienne, Yémen, Djibouti, Iran) et cinq pays de celles de l'Afrique du Nord (Tunisie, Algérie, Egypte, Maroc, Mauritanie). En considérant la région MENA, le choix des pays s'est fait en fonction de la disponibilité des données et de manière à obtenir des séries qui contiennent des pays surendettés afin de déterminer l'effet du fardeau de la dette, et des pays moyennement endettés, dont on peut montrer l'effet d'une dette modérée sur la croissance économique.

En considérant des données annuelles, la période d'étude couvre 21 ans et s'étale entre 1990 et 2010.

Les déterminants types de la croissance sur lesquels se base cette étude sont les suivants.

- Le taux de croissance du PIB réel par habitant décalé d'une période (TCPIBHR) : cette variable peut avoir une grande influence sur la croissance et permet de vérifier l'hypothèse de convergence non conditionnelle au sein d'un groupe de pays. L'hypothèse de convergence conditionnelle implique que le coefficient de cette variable soit significativement négatif. Dans ce cas, les variables de contrôle et l'effet spécifique individuel saisissent le niveau de revenu de long terme vers lequel chaque pays converge.

- Le taux de croissance de la population (TCD) : Une augmentation de la population pourrait influencer négativement le taux de croissance économique. Autrement dit, une croissance démographique élevée tend à appauvrir un pays dans la mesure où il est difficile de préserver un volume important de capital par travailleur en présence d'une croissance rapide du nombre de travailleurs (Mankiw 2003).

- Le taux d'investissement (TINV) : il indique la part de l'investissement total dans le PIB et reflète l'impact du facteur capital physique dans le processus de production. Considéré comme une source de croissance, cette variable permet d'accroître le capital physique, d'augmenter la production, et par conséquent, le revenu.

- le taux de scolarisation au secondaire (DCH): il mesure le développement du capital humain, qui permet d'augmenter la productivité des travailleurs, et donc leur revenu, de même que celui de l'économie nationale.

- Le degré d'ouverture d'un pays (OUV) : il s'agit d'un indicateur de l'ouverture commerciale. La plupart des études (théoriques et empiriques) affirment que plus un pays était ouvert sur l'extérieur, plus cela lui permettrait de stimuler sa productivité et de réorienter ses ressources rares vers des secteurs plus efficients et donc d'améliorer son bien-être.

- Les ratios d'endettement : il s'agit du service de la dette extérieure en pourcentage des exportations (SDEXP) et de la valeur nominale de l'encours de la dette publique extérieure, exprimée en pourcentage du PIB (DETPIB) et en pourcentage des exportations de biens et de services (DETEXP). La dette extérieure est destinée à combler le déficit de ressources internes nécessaire au financement de la croissance. En effet, l'entrée des capitaux étrangers permet de renforcer la capacité d'investissement et provoquer ainsi un accroissement de la richesse nationale. Cependant, son accroissement signifie que la dette totale croît plus rapidement que les sources fondamentales du revenu.

Comme précédemment indiqué, l'introduction de ces variables permet de vérifier deux hypothèses. La première est l'existence d'une relation non linéaire entre la dette et la croissance tandis que la seconde est que le paiement futur de la dette décrit par son encours en augmentant la probabilité d'un accroissement de la pression fiscale, décourage les investissements domestiques et étrangers.

Pour le service de la dette en pourcentage des recettes d'exportation, il montre la vulnérabilité des engagements dans laquelle le paiement des charges au titre du service de la dette, est exposé au risque d'une baisse inattendue des recettes d'exportation.

Nous notons ici que dans le but de faire la distinction entre l'effet d'éviction de la dette et la thèse du surendettement (l'existence d'une relation entre la courbe de Laffer et la croissance), nous avons utilisé le service de la dette rapporté aux exportations et les indicateurs de la dette au premier et second degré. En théorie, le service de la dette extérieure (qui comprend les intérêts et le remboursement du principal) peut influer sur la croissance en évinçant les investissements privés ou en modifiant la composition des dépenses publiques. L'augmentation des charges d'intérêts peut accroître le déficit budgétaire du pays et réduire ainsi l'épargne publique, ce qui entraîner soit une hausse des taux d'intérêt, soit l'éviction des investissements privés, freinant ainsi la croissance économique. Par ailleurs, l'accroissement du service de la dette peut avoir un effet négatif sur la croissance en réduisant le montant des ressources publiques disponibles pour les infrastructures et la formation du capital humain. 
Dans nos estimations, le ratio service de la dette par rapport aux exportations doit avoir un signe négatif pour illustrer cet effet d'éviction tandis que pour l'existence de la thèse du surendettement, il faut que le signe du coefficient de la dette soit positif et celui de la dette au carré négatif. Donc, le pic de l'équation quadratique va identifier le niveau du stock de la dette où l'impact marginal de la dette sur la croissance devient négatif.

- Variation des termes de l'échange (VTE) : cette variable permet de capter les effets des chocs extérieurs dans ces économies surtout que la plupart des pays considérés est dépendante et exportatrice des matières premières.

- Taux d'inflation (TINF) : La majorité des travaux empiriques considère que cette variable influence négativement la croissance réelle. En effet, l'inflation accroît le coût du capital, ce qui réduit les investissements et par conséquent, la croissance économique. Par ailleurs, l'introduction de cette variable dans l'équation de croissance permet d'apparaître l'effet d'éviction du service de la dette (Agénor et Montiel, 1999). Un service de dette élevé peut inciter les gouvernements à adopter des politiques inflationnistes ce qui affecte négativement l'investissement et par conséquent, la croissance économique. Le tableau ci-après récapitule toutes les variables utilisées ainsi que leurs sources statistiques.

Tableau 1. Liste des variables du modèle

\begin{tabular}{|c|c|c|c|}
\hline Variables explicatives & Notation & Signe attendu & $\begin{array}{c}\text { Sources } \\
\text { statistiques }\end{array}$ \\
\hline Taux de croissance du PIB réel par habitant & TCPIBRH & $* * * * *$ & \multirow{11}{*}{$\begin{array}{l}\text { WDI, Banque } \\
\text { Mondiale, } \\
2012\end{array}$} \\
\hline Taux de croissance du PIB réel par habitant décalé & TCPIBHR & négatif & \\
\hline Taux de croissance démographique & TCD & négatif & \\
\hline $\begin{array}{l}\text { Taux d'investissement brut (public et privé), en } \\
\text { pourcentage du PIB }\end{array}$ & TINV & positif & \\
\hline Taux de scolarisation dans le secondaire & $\mathrm{DCH}$ & positif & \\
\hline Variation des termes de l'échange & VTE & positif & \\
\hline Ouverture commerciale & OUV & positif & \\
\hline $\begin{array}{l}\text { Total du service de la dette (publique et privée), en } \\
\text { pourcentage des recettes d'exploitation }\end{array}$ & SDEXP & négatif & \\
\hline Dette extérieure en pourcentage de PIB & DETPIB & positif & \\
\hline $\begin{array}{l}\text { Dette extérieure en pourcentage des exportations } \\
\text { des biens et services }\end{array}$ & DETEXP & positif & \\
\hline Taux d'inflation & TINF & négatif & \\
\hline
\end{tabular}

\subsection{Interprétation des résultats}

\subsubsection{Statistiques descriptives et matrice des corrélations}

Le tableau 2 de l'annexe présente une analyse descriptive des variables explicatives et de la variable endogène. Nous constatons que ces variables ne suivent pas la loi normale puisque les statistiques de Jarque \& Berra sont supérieures à la valeur critique de Khi-deux à deux degrés de libertés. Aussi, ces variables sont asymétriques puisque les statistiques de Kurtosis sont supérieures à trois (sauf pour la variable $\mathrm{DCH}$ ) et ne possèdent pas des branches paraboliques de directions asymptotiques vers l'axe d'abscisse.

La matrice de corrélation permet de vérifier l'existence ou non d'un problème de multicolinéarité. Les résultats trouvés sont présentés dans le tableau 3 de l'annexe, qui nous montre que les coefficients de corrélation sont faibles pour la plupart des variables retenues. Toutefois, nous remarquons qu'il existe des coefficients élevés de corrélation pour les variables dette extérieure en pourcentage des exportations des biens et services (DETEXP) et dette extérieure en 
pourcentage du PIB (DETPIB). Cependant, ces deux variables ne seront pas retenues conjointement dans la régression. En somme, nous pouvons conclure qu'il y a absence d'un problème de multicolinéarité.

\subsubsection{Stationnarité des séries}

L'étude de la stationnarité des variables permet d'avoir une idée sur les caractéristiques des séries étudiées. En se basant sur les résultats du tableau 4 de l'annexe, nous pouvons constater que toutes les variables sont stationnaires en niveau puisque les valeurs calculées des statistiques du test de Levin, Lin et Chu (LLC ; 2002) sont inférieures à la valeur critique de la loi normale centrée réduite au seuil du risque de $5 \%(-1,64)$.

Ainsi, notre premier intérêt est de déterminer les tests de spécification ou tests d'homogénéité des données. Nous allons montrer si le modèle considéré est parfaitement identique pour tous les pays de l'échantillon ou bien s'il y a des spécificités propres à chaque pays.

En fait, les résultats tirés des statistiques de Fisher (tableaux 5 et 6 de l'annexe) montrent le rejet de l'hypothèse d'homogénéité global sachant qu'il existe des coefficients communs pour tous les pays ainsi que la présence des spécificités individuelles pour chaque pays ( $P$-Value $<10 \%$ ). Par conséquent, nous pouvons conclure que notre modèle est spécifié par un panel avec des effets individuels. La question qui se pose maintenant est de savoir quel type d'effet individuel à retenir? Pour répondre à cette interrogation, nous utiliserons le test d'Hausman (1978). De même, pour faire la distinction entre les deux techniques d'estimation, Within ou GLS, nous adopterons ce test de spécification d'Hausman.

D'après les tableaux 3 et 4 , nous pouvons affirmer que notre modèle est spécifié par un panel avec des effets individuels fixes puisque les statistiques d'Hausman sont supérieures à la valeur critique de Khi-deux à neuf degrés de libertés $\left(\chi^{2}(9)=14,68\right.$ Pour $\alpha=10 \%$ ). D'où, l'estimation avec Within (estimateur non biaisé) est la plus appropriée.

\subsubsection{Interprétation des résultats}

Conformément à ce que prédit la littérature sur la croissance économique, le coefficient du taux de croissance démographique a bien le signe négatif attendu mais pour la plupart des estimations (linéaires et quadratiques) ce ratio n'est pas significatif (les tableaux 5 et 6 de l'annexe). Ceci corrobore les résultats relevés par plusieurs travaux comme ceux d'Ojo et Oshikoya (1995).

Pour les coefficients associés à la variable investissement, ils sont positifs pour l'estimation Within et statistiquement significatifs (tableaux 4 et 5 de l'annexe). Ceci confirme la théorie selon laquelle le développement d'un pays passe avant tout par l'effort consenti en matière d'investissements productifs.

Concernant le développement du capital humain, il exerce un impact positif et significatif sur le taux de croissance économique dans la majorité des estimations linéaires et quadratiques (pour l'estimation avec des effets fixes). Ceci justifie le fait que l'éducation est souvent considérée comme la clef de voûte du développement économique. Toutefois, il faut être prudent avec cet indicateur, car il ne tient pas compte de la qualification, donc de l'efficacité.

Dans la plupart des estimations effectuées, les variations des termes de l'échange exercent un impact positif mais non significatif sur la croissance économique.

Le taux d'ouverture commerciale contribue positivement et significativement à la croissance économique (tableaux 5 et 6 ) pour la méthode Within. En effet, l'ouverture économique permet à l'économie de bénéficier des transferts technologiques et des différentes formes d'externalités positives et des effets d'entraînements de la demande extérieure. Egalement, l'ouverture sur l'extérieure permet aux pays d'obtenir, en cas de besoin, de meilleures conditions d'emprunt sur le marché international. D'autre part, la compétitivité internationale d'un pays est tributaire à une plus grande ouverture commerciale, exigeant un accroissement de la productivité des facteurs, une promotion des activités à haute valeur ajoutée et à vocation exportatrice ainsi qu'un assouplissement des conditions de fonctionnement du marché du travail. La diversification ainsi que la meilleure rémunération des exportations sont également une condition primordiale à la compétitivité.

Considéré comme un indicateur primordial de la qualité de l'environnement économique, le taux d'inflation a été introduit dans l'équation de croissance pour faire apparaître l'effet d'éviction du service de la dette. Un service de dette élevé incite souvent les gouvernements à adopter des politiques inflationnistes qui peuvent affecter négativement l'investissement et, par conséquent, la croissance économique (Agénor et Montiel (1999)). Les estimations font ressortir des coefficients du taux d'inflation négatifs et statistiquement significatifs ce qui est conforme aux résultats attendus par la théorie économique.

Une augmentation du service de la dette a un impact négatif et significatif sur la croissance économique pour l'ensemble des estimations effectuées. Ainsi et comme on a déjà mentionné, ce ratio d'endettement mesure la proportion du service de la dette extérieure couverte par le produit intérieur brut. II s'agit d'un indicateur fortement utile car il permet de surveiller 
le niveau d'endettement du pays. Lorsqu'il est élevé, il pourrait empêcher un pays de consacrer des ressources suffisantes aux activités productives. Les résultats obtenus corroborent les travaux de Pattillo et al. (2002), qui ont montré que l'impact de cette variable sur la croissance économique, est soit non significatif, soit négatif et significatif. Donc, le service de la dette affecte la croissance à travers son impact sur la qualité de l'investissement ou bien à travers le volume élevé de la dette.

En appliquant le modèle linéaire, les résultats relevés indiquent que la dette extérieure rapportée successivement aux exportations de biens et services et au PIB influence positivement et significativement la croissance conformément à ce que prédit la théorie économique. Ainsi, considéré comme un instrument de financement extérieur destiné à l'investissement, l'endettement extérieur en soit n'est pas un frein au développement dans ces pays soufrant souvent d'une carence de ressources internes. Comme le souligne plusieurs travaux empiriques, ce n'est lorsque cet endettement extérieur atteint un certain seuil qu'il devient un handicap pour la croissance (Patillo et al (2002)). A partir d'un tel niveau d'endettement, l'efficacité marginale d'une unité monétaire empruntée devient inférieure au taux d'intérêt appliqué pour le remboursement de la dette.

Concernant l'estimation du modèle quadratique, il a été effectué pour tester l'hypothèse des rendements marginaux décroissants de l'endettement extérieur, c'est-à-dire l'effet non linéaire de la dette extérieure sur la croissance économique. Dans la lignée des travaux de Lensink et White (1999), Hansen et Tarp (2000, 2001), Dalgaard et Hansen (2001), Collier et Dollar $(2001,2002)$ et Patillo et al (2002), nous avons introduit dans les estimations du modèle de croissance les indicateurs de la dette relevés au carré (tableaux 5 et 6 ).

Les résultats obtenus s'inscrivent bien dans la prédiction théorique. En effet, les coefficients des deux variables d'endettement retenus au carré (dette extérieure en pourcentage du PIB et dette extérieure en pourcentage des exportations) sont négatifs bien que leur significativité reste faible. Autrement dit, la dette extérieure affecte positivement la croissance si elle ne dépasse pas un certain seuil. Au delà de ce seuil, son effet devient négatif. Ceci nous permet d'opter pour l'existence d'une relation non linéaire entre la dette et la croissance, c'est-à-dire qu'il y a un seuil critique qui rendrait la croissance négative.

Pour illustrer le lien entre les investissements et le service de la dette (effet d'éviction), nous avons estimé le modèle de croissance sans investissement (tableaux 5 et 6). Ceci nous permet de vérifier l'hypothèse selon laquelle une partie de l'effet de la dette soit incorporée dans un ratio plus faible d'investissement, ce qui peut biaiser les résultats des estimations. Ainsi, lorsque l'effet de la dette est beaucoup plus important quand l'investissement est exclu de l'estimation, nous pouvons conclure qu'une dette élevée réduit le taux d'investissement. Dans le cas contraire, c'est-à-dire lorsque cet écart est négligeable, on peut déduire que le canal essentiel par lequel la dette affecte la croissance économique est plutôt la qualité et l'efficacité de l'investissement (Pattillo et al., 2002).

En tenant compte de cette explication, les estimations effectuées montrent que les coefficients liés aux variables d'endettement (DETPIB, DETEXP) sont significatifs et au même temps sont différents de ceux obtenus en présence d'investissement. Autrement dit, le ratio du service de la dette reste significatif signalant que son effet indésirable passe par l'effet d'éviction, mais aussi affecte directement le taux de croissance économique.

Les résultats ainsi dégagés nous permettent de conclure que la théorie de surendettement est bien vérifiée. II existe donc, conformément aux résultats de Clement et al (2003), une relation entre la dette et la croissance qui prend la forme d'un $U$ inversé, indiquant l'existence d'une courbe de Laffer de la dette.

En utilisant la méthode quadratique, nous avons déterminé le seuil d'endettement optimal qui correspond à l'impact marginal de la dette. Ce seuil correspond au niveau d'endettement optimal qui maximise la croissance économique (Pattillo et al. 2002). Nous l'avons déterminé en résolvant la formule suivante $\exp \left(-\frac{\beta_{\mathrm{D}}}{2 \beta_{\mathrm{D} 2}}\right)$.

Table 2 : Seuil d'endettement ou point de détournement de la dette

\begin{tabular}{|c|c|}
\hline Indicateur d'endettement & $\begin{array}{l}\text { Seuil d'endettement ou point } \\
\text { de détournement de la dette }\end{array} e^{-\left(\frac{\beta_{D}}{2 \beta_{D 2}}\right)}$ \\
\hline DETEXP & $59,14 \%$ \\
\hline DETPIB & $28,03 \%$ \\
\hline
\end{tabular}


Le tableau 2 montre le niveau de la dette au-delà duquel l'impact marginal de la dette extérieure sur la croissance économique devient négatif. Le seuil d'endettement optimal varie entre $28,03 \%$ des exportations et $59,14 \%$ du PIB pour les pays d'Afrique de Nord.

\section{CONCLUSION}

L'objet de la présente étude est d'évaluer l'impact de la dette extérieure sur le développement économique (à travers la croissance) pour un panel de pays en développement. Les résultats obtenus sur l'échantillon total des pays montrent que l'impact de la dette extérieure sur le niveau de croissance économique s'inscrit bien dans les prédictions théoriques. Nous avons retenu les principales conclusions suivantes.

i. Quelque soit le type du modèle considéré, linéaire ou quadratique, le taux de croissance démographique et le taux d'inflation exercent un effet négatif et significatif sur le taux de croissance de l'économie ;

ii. Les variables taux d'investissement, croissance du capital humain, variations des termes de l'échange et taux d'ouverture commercial interviennent positivement dans le processus de développement économique ;

iii. Une augmentation du service de la dette a un impact négatif et significatif sur la croissance économique. Le canal de transmission de cet impact néfaste passe par la qualité de l'investissement et par le fardeau de la dette ;

iv. En appliquant le modèle linéaire, les estimations relevées montrent que les deux variables de l'endettement extérieur (DETPIB et DETEXP) exercent un effet positif sur le taux de croissance de l'économie. Ceci implique que la dette extérieure en soit n'est pas un frein au développement dans ces pays qui soufrant souvent d'une carence de ressources internes.

v. L'application du modèle quadratique a été faite afin de tester l'hypothèse des rendements marginaux décroissants de l'endettement extérieur ; autrement dit l'effet non linéaire de la dette extérieure sur la croissance économique. Les résultats obtenus indiquent que la dette extérieure affecte positivement la croissance si elle ne dépasse pas un certain seuil. Au delà de ce seuil, son effet devient négatif. Nous pouvons, en conséquence, admettre l'existence d'une relation non linéaire entre la dette et la croissance, c'est-à-dire qu'il y a un seuil critique qui rendrait la croissance négative. Ce seuil, qui correspond à l'impact marginal de la dette, varie entre $28,03 \%$ des exportations de biens et services et $59,14 \%$ du PIB pour les pays de la région MENA.

vi. L'estimation du modèle de croissance sans investissement a été effectuée dans le but de vérifier l'hypothèse de l'effet d'éviction. Les résultats trouvés montrent que les coefficients liés aux variables d'endettement sont à la fois significatifs et différents de ceux obtenus en présence d'investissement. Autrement dit, le ratio du service de la dette reste significatif ce qui indique que son effet indésirable passe par l'effet d'éviction, mais aussi affecte directement le taux de croissance économique. Ce résultat fort intéressant nous permet de conclure que la théorie de surendettement est bien vérifiée. II existe donc une relation entre la dette et la croissance qui prend la forme d'un $U$ inversé, indiquant la présence d'une courbe de Laffer de la dette.

\section{REFERENCES}

[1] Adam, C. S. and D. L. Bevan (2005), «Fiscal deficits and growth in developing countries», Journal of Public Economics», Vol. (4), pp. 571-597. al Folly, Princeton University Press.

[2] Agenor, P. R. et P. J. Montiel (1999), « Development macroeconomics », Banque du Mexique (2004) : Rapport annuel.

[3] Ardagna, S., F. Caselli and T. Lane (2007) «Fiscal Discipline and the Cost of Public Debt Service: Some Estimates for OECD Countries», the B.E. Journal of Macroeconomics: Vol. 7: Iss. 1 (Topics), Article 28.

[4] Arslanalp, S et Henry, P.B. (2006), « Debt relief », BER Working Paper Series N¹21.

[5] Asiedu, E (2003), «Debt relief and institutional reform: a focus on Heavily Indebted Poor Countries », The Quarterly Review of Economics and Finance, 43. 614-626.

[6] Barro Robert J. et Xavier Sala-i-Martin (1995), Economic Growth, McGraw-Hill, Inc.

[7] Bulow J., Rogoff K. (1990), «Cleaning up third world debt without getting taken to the cleaners», Journal of Economic Perspectives, vol. 4, $\mathrm{n}^{\circ} 1$, p. 31-42.

[8] Carmen M. Reinhart \& Kenneth S. Rogoff, (2009), «This Time is Different: Eight-Centuries of Financial Folly », Princeton, NJ, Princeton University Press.

[9] Carmen M. Reinhart., \& Kenneth S. Rogoff (2010), « Growth in a time of debt », in_American Economic Review 2 vol. $100, n^{\circ} 2$, mai. 
[10] Cecchetti, Stephen G., M. S. Mohanty et Fabrizo Zampolli (2011), «The real effects of debt», BIS Working paper, n`352, août.

[11] Checherita C. and P. Rother (2010), «the impact of high and growing government debt on economic growth: An empirical investigation for the euro area», ECB, Working Paper 1237, August.

[12] Claessens, S et I, Diwan (1989): «Liquidity, debt relief, and conditionality », in « Dealing with the debt crisis ». 213228. World Bank.

[13] Clemens Benedict J., Rina B. et Toan Quoc N. (2003), «External Debt, Public Investment and Growth in Lowincomes countries ", IMF Working Paper, WP/03/249.

[14] Cohen D. (1995), «Large External Debt and (Slow) Domestic Growth», Journal of Economic Dynamics and Control, Vol. 19, pp. 1141- 1163.

[15] Cohen Daniel (1993), «Low Investment and Large LDC Debt in the 1980's », American Economic Review, juin, pp. 436-449.

[16] Cohen, D. (1997), "Growth and external debt: A new perspective on the African and Latin American tragedies», Centre for Economic Policy Research Discussion Paper No. 1753.

[17] Collier, P. and D. Dollar (2001) «Can the World Cut Poverty in Half? How Policy Reform and Effective Aid Can Meet the International Development Goals», World Development, 29 (11), pp. 1787-1802.

[18] Collier, P. and D. Dollar (2002) «Aid Allocation and Poverty Reduction », European Economic Review, 45, pp. 14701500.

[19] Cordella, T., Ricci, L.A et Ruiz-Arranz, M (2005), «Debt overhang or debt irrelevance? Revisiting the debt-growth link ». IMF Working Paper. (WP/05/223).

[20] Corden (D) W. M., «Debt relief and Adjustment incentives», IMF Staff Papers, 35(4), pp 628-643.

[21] Corsetti G., K. Kuester, A. Meier and G. Mueller (2010), «Debt consolidation and fiscal stabilization of deep recessions », American Economic Review, 100 (2), 41-45, May.

[22] Dalgaard C.J. et Hansen H. (2001), « On Aid Growth and Good Policies », Journal of Development Studies, vol. 37(6), pp.17-41.

[23] De Prest E., B. Eugène and L. Van Meensel (2010), «Strategies and measures aimed at consolidating public finances », NBB, Economic Review, 43-63, September.

[24] Depetris Chauvin, N et Kraay, A (2005), «What has 100 billion dollars worth of debt relief done for low-income countries? ». Economics Working Paper Archive, № 0510001.

[25] Deshpande, A (1997), «The debt overhang and the disincentive to invest », Journal of Development Economics, 52 (1). 169-187.

[26] Diamond, P. (1965), «National Debt in a Neoclassical Growth Model», American Economic Review, 55 (5), pp. 1126 1150.

[27] Dijkstra G., Hermens N. (2001), «The Uncertainty of Debt Service Payments and Economic Growth of Highly Indebted Poor Countries: Is There a Case for Debt Relief ?» UNU/ WIDER Development Conference on Debt Relief, 17- 18 août, Helsinki, Finlande.

[28] Easterly William (2002), «Growth implosions, debt explosions and my Aunt Marilyn: Do growth slowdowns cause public debt crisis ?» World Bank policy research Working Paper, n²531.

[29] Elmendorf D. W. et N. G. Mankiw (1999), « Government debt, in Taylor J.B. and M. Woodford », eds, Handbook of Macroeconomics, Amsterdam, Elsevier Science, B.V., vol. 1, pp. 1615-1699.

[30] Ferreira C. (2009), «Public Debt and Economic Growth: a Granger Causality Panel Data Approach», Université de Lisbonne, Working paper n²4/2009/DE/UECE.

[31] Hansen Henrik (2001), «The impact of aid and external debt on growth and investment: Insights from cross- country regressions analysis», WIDER conference on debt relief, Helsinki, 17-18 august, 21p. 
[32] Hansen, H. et F. Tarp (2000), « Aid Effectiveness Disputed », Journal of International Development, 12(3), pp.37598.

[33] Hansen, H. et F. Tarp (2001), «Aid and Growth Regressions », Journal of Development Economics, 64 (2), 547-70.

[34] Harrison A. (1996), "Openness and Growth, A Time-series, Cross-Country Analysis for Developing Countries», Journal of Development Economics,vol. 48, no 2, pp.419-447.

[35] Klein, T.M. (1994), «External debt Management», World Bank Technical paper N²45, Washington DC.

[36] Krugman P. (1988a), «Financing vs. Forgiving a Debt Overhang», NBER Working Paper n²486, Janvier 1988.

(37] Kumar, M. and J. Woo (2010), «Public Debt and Growth», IMF Working Paper 10/174.

[38] Lensink R., White H. (1999), «Is There an Aid Laffer Curve? », CREDIT Research Paper n 99/6, Centre for Research in Economic Development and Institutional Trade, University of Nottingham.

[40] Levin A., Lin C.F. et Chu C.S.J. (2002), « Unit Root Test in Panel Data: Asymptotic and Finite Sample Properties », Journal of Econometrics, 108, 1-24.

[41] Maghyereh A. I, Omet G., Kalaji F. (2002), «External Debt and Economic Growth in Jordan: The Threshold Effect », The Hachemite University. Jordanie.

[42] Mankiw N. G. 2003. Macroéconomie, édition De Boeck, France, 655 pages.

[43] Mankiw N.G., Romer D. et Weil D.N. (1992), "A Contribution to the Empirics of Economic Growth », Quarterly Journal of Economics, vol. 107 (2), pp. 407-437.

[44] Nautet, M., et L. Van Meensel (2011) « Economic impact of the public debt », in_Economic Review, $n^{\circ} 3$.

[45] Nersisyan, Yeva, \& L. Randall Wray (2011), « Un excès de dette publique handicape-t-il réellement la croissance ? », in Revue de l'OFCE, n 116, janvier 2011.

[46) Panizza, Ugo, et Andrea F Presbitero (2012),_“ Public Debt and Economic Growth: Is There a Causal Effect? », MoFiR working paper, $n^{\circ} 65,2$ avril.

[47] Patillo, C., Poirson, H., et Ricci, L. (2002), « External debt and growth ». IMF Working Paper (WP/02/69).

[48] Pattillo C., Poirson H. et Ricci L. (2004), «What Are the Channels Through Which External Debt Affects Growth? », IMF Working Paper, $\mathrm{n}^{\circ}$. 04/15. IMF, Washington, DC.

[49] Presbitero, A. F (2009), « Debt-relief effectiveness and institution-building », Development Policy Review, 27(5). 529559.

[50] Reinhart, C. M. and K. S. Rogoff (2009), «The Aftermath of Financial Crisis», American Economic Review, Vol. 99(2), pp. 466-472.

[51] Reinhart, C. M. and K. S. Rogoff (2010), «Growth in a Time of Debt», NBER Working Paper No. 15639.

[52] Clemens et al. (2003), «External Debt, Public Investment and Growth in Low-income countries », IMF, Working Paper, WP/03/249.

[53] Ricciuti R., (2003), « Assessing ricardian equivalence », Journal of Economic Surveys, 17(1), février, pp. 55-78.

[54] Sen, S., Kasibhalta, K. M., et Stewart, D.B (2007), « Debt overhang and economic growth -the Asian and the Latin American experiences », Economic systems, 31. 3-11.

[55] Sevestre P (2002), Econométrie des données de Panel, Dunod, Paris.

[56] Swapan S., Kasibhatla M. et Stewart D.B. (2007), « Debt overhang and economic growth: the Asian and the Latin American experiences », Economic Systems, vol. 31, pp 3-11.

[57] Warner A.M. (1996), « Did the Debt Crisis Cause the Investment Crisis? », Quaterly Journal of Economics, vol. 107, $\mathrm{n}^{\circ} 4$, November. 
Annexes

Tableau 2 : Statistiques Descriptives

\begin{tabular}{|c|c|c|c|c|c|c|c|c|c|c|c|}
\hline & TCPIBH & TCPIBHR & TCD & TINV & $\overline{\mathrm{DCH}}$ & VTE & OUV & SDEXP & DETEXP & DETPIB & TINF \\
\hline Mean & 2.150571 & 1.737607 & 2.175859 & 23.25375 & 57.22970 & -4117884 & 0.753074 & 16.08679 & 226.1755 & 66.05394 & 9.443723 \\
\hline Median & 2.159605 & 2.024662 & 1.900677 & 23.01550 & 61.13682 & 0.003660 & 0.693559 & 13.30003 & 167.8234 & 55.74391 & 5.167508 \\
\hline Maximum & 34.65264 & 34.65264 & 11.18066 & 58.95761 & 95.43458 & 1.669202 & 1.651394 & 83.57682 & 994.9874 & 207.7975 & 119.9893 \\
\hline Minimum & -8.947317 & -42.80901 & 0.590165 & 6.112138 & 10.41678 & -45100630 & 0.293684 & 1.045315 & 7.230255 & 3.257153 & -3.846154 \\
\hline Std. Dev. & 4.394641 & 5.530536 & 1.071283 & 6.825023 & 24.43025 & 13019183 & 0.256804 & 13.28791 & 180.3062 & 45.63083 & 12.72978 \\
\hline Skewness & 2.164689 & -1.299769 & 3.088213 & 0.911002 & -0.388039 & -2.837756 & 0.780850 & 1.898633 & 1.618131 & 1.099199 & 4.261337 \\
\hline Kurtosis & 18.12441 & 26.73270 & 23.79315 & 6.890876 & 1.912417 & 9.052859 & 3.357716 & 8.430946 & 6.043447 & 3.808829 & 30.35116 \\
\hline Jarque-Bera & 2371.792 & 5462.486 & 4508.992 & 176.8950 & 17.10754 & 659.7986 & 24.59914 & 420.8463 & 189.1363 & 52.58527 & 7865.254 \\
\hline Probability & 0.000000 & 0.000000 & 0.000000 & 0.000000 & 0.000193 & 0.000000 & 0.000005 & 0.000000 & 0.000000 & 0.000000 & 0.000000 \\
\hline Sum & 494.6314 & 399.6497 & 500.4476 & 5348.363 & 13162.83 & $-9.47 E+08$ & 173.2071 & 3699.962 & 52020.36 & 15192.41 & 2172.056 \\
\hline Sum Sq. Dev. & 4422.648 & 7004.384 & 262.8112 & 10667.04 & 136675.7 & $3.88 \mathrm{E}+16$ & 15.10220 & 40434.20 & 7444866. & 476817.6 & 37108.84 \\
\hline Observations & 230 & 230 & 230 & 230 & 230 & 230 & 230 & 230 & 230 & 230 & 230 \\
\hline Cross sections & 11 & 11 & 11 & 11 & 11 & 11 & 11 & 11 & 11 & 11 & 11 \\
\hline
\end{tabular}


Tableau 3 : Matrice des corrélations

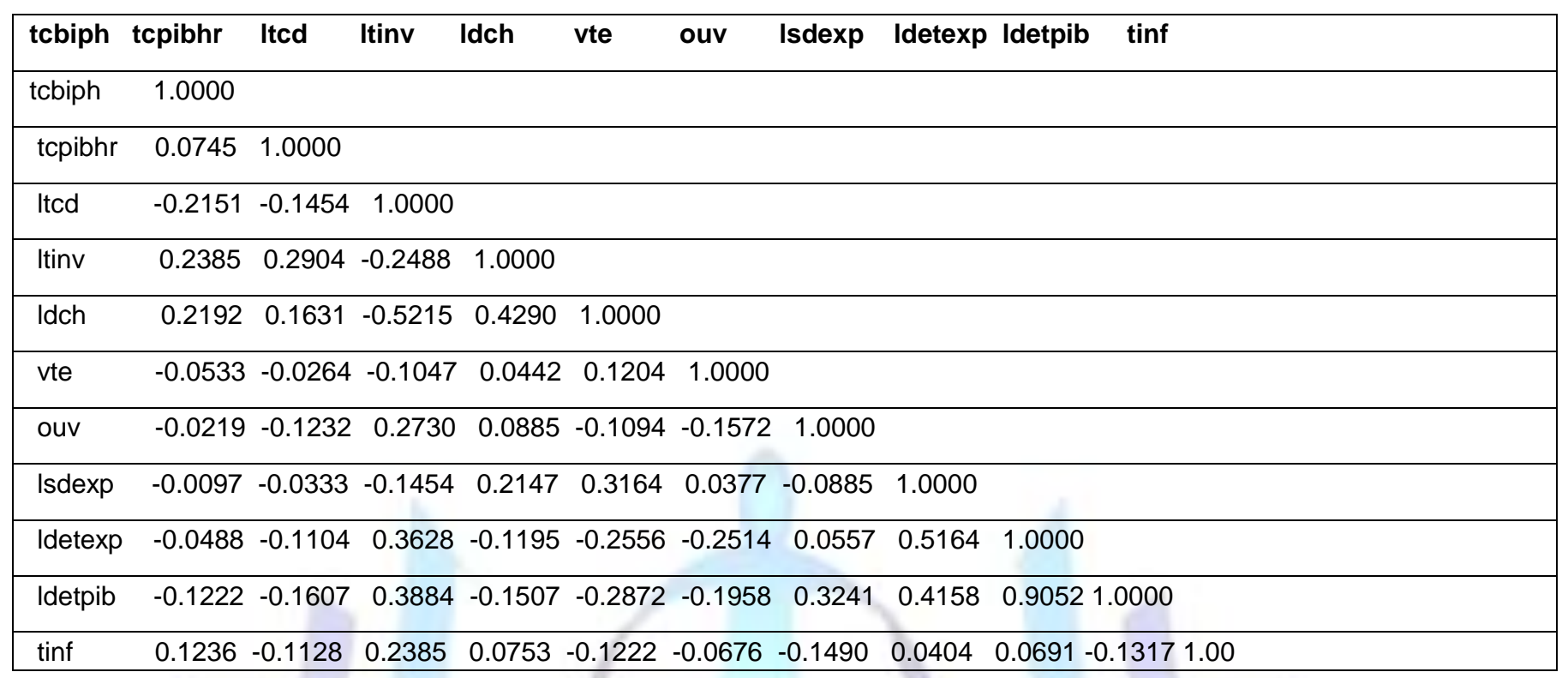

Tableau 4 : Statistiques de stationnarité de LLC (2002)

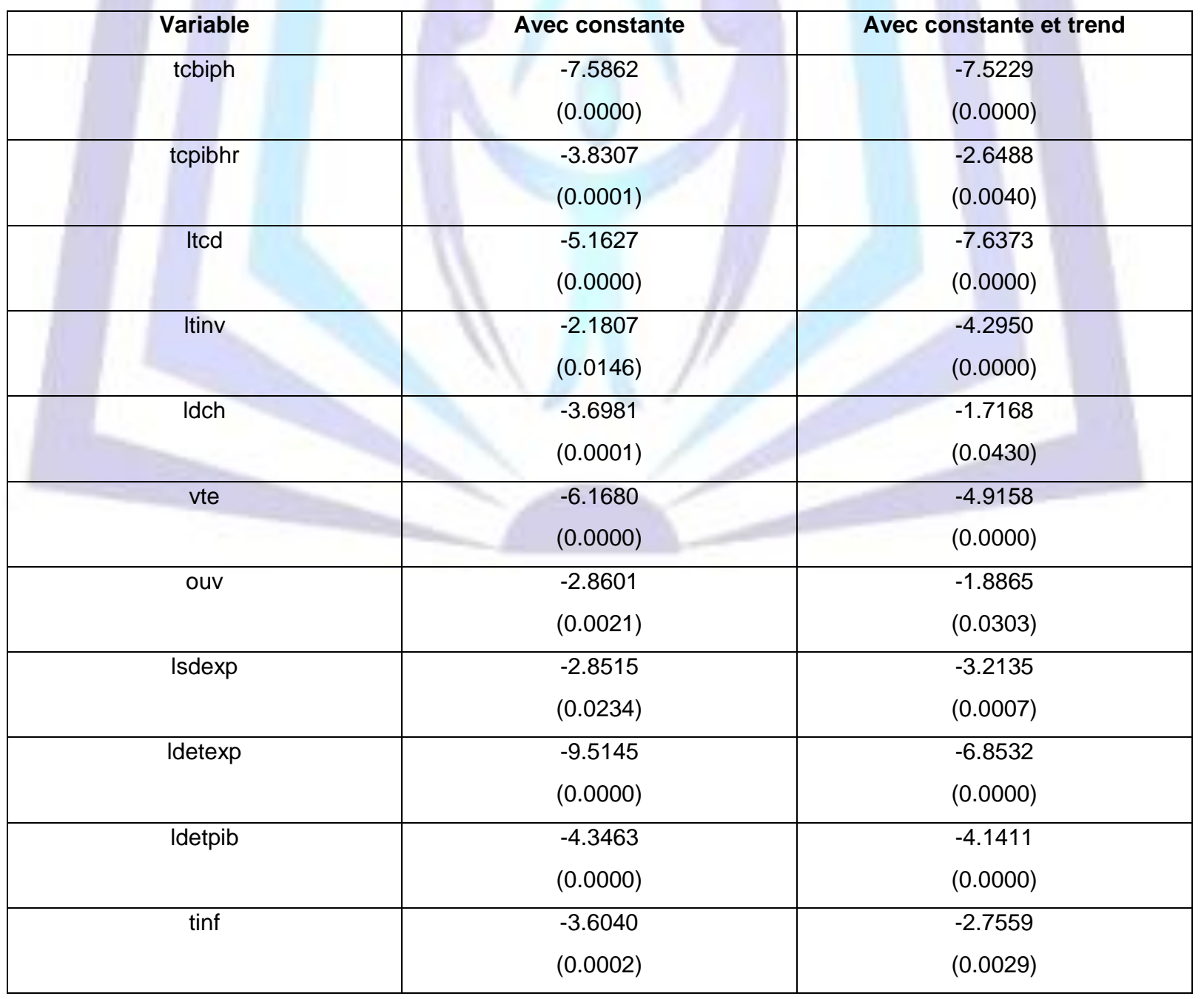


Tableau 5 : Résultats des estimations du modèle linéaire et quadratique Indicateur d'endettement : dette extérieure en pourcentage des exportations Variable dépendante : taux de croissance du PIB réel par habitant

\begin{tabular}{|c|c|c|c|c|c|c|c|c|}
\hline \multirow{3}{*}{ Variables } & \multicolumn{4}{|c|}{ Avec Investissement } & \multicolumn{4}{|c|}{ Sans Investissement } \\
\hline & \multicolumn{2}{|c|}{ Linéaire } & \multicolumn{2}{|c|}{ quadratique } & \multicolumn{2}{|c|}{ Linéaire } & \multicolumn{2}{|c|}{ quadratique } \\
\hline & Within & GLS & Within & GLS & Within & GLS & Within & GLS \\
\hline Tcpibt-1 & $\begin{array}{c}-0.02 \\
(-0.41)\end{array}$ & $\begin{array}{l}0.003 \\
(0.06)\end{array}$ & $\begin{array}{c}-0.02 \\
(-0.52)\end{array}$ & $\begin{array}{l}-0.001 \\
(-0.02)\end{array}$ & $\begin{array}{c}-0.02 \\
(-0.52)\end{array}$ & $\begin{array}{c}0.03 \\
(0.73)\end{array}$ & $\begin{array}{c}-0.03 \\
(-0.62)\end{array}$ & $\begin{array}{c}0.03 \\
(0.71)\end{array}$ \\
\hline LTCD & $\begin{array}{c}-1.61 \\
(-1.27)^{* * *}\end{array}$ & $\begin{array}{c}-2.67 \\
(-3.06)^{*}\end{array}$ & $\begin{array}{c}-1.46 \\
(-1.14)^{\star \star \star}\end{array}$ & $\begin{array}{c}-2.56 \\
(-2.88)^{*}\end{array}$ & $\begin{array}{c}-1.69 \\
(-1.35)^{* \star *}\end{array}$ & $\begin{array}{c}-2.85 \\
(-3.26)^{*}\end{array}$ & $\begin{array}{c}-1.53 \\
(-1.20)^{\star \star \star}\end{array}$ & $\begin{array}{c}-2.81 \\
(-3.83)^{*}\end{array}$ \\
\hline LTINV & $\begin{array}{c}2.22 \\
(2.08)^{\star}\end{array}$ & $\begin{array}{c}0.55 \\
(0.39)\end{array}$ & $\begin{array}{c}2.33 \\
(2.15)^{*}\end{array}$ & $\begin{array}{c}0.49 \\
(0.36)\end{array}$ & & & & \\
\hline $\mathrm{LDCH}$ & $\begin{array}{c}3.19 \\
(1.57)^{* * *}\end{array}$ & $\begin{array}{c}0.82 \\
(2.21)^{*}\end{array}$ & $\begin{array}{c}3.39 \\
(1.65)^{\text {** }}\end{array}$ & $\begin{array}{c}0.85 \\
(2.25)^{*}\end{array}$ & $\begin{array}{c}2.79 \\
(1.59)^{* * *}\end{array}$ & $\begin{array}{c}1.03 \\
(2.85)^{*}\end{array}$ & $\begin{array}{c}3.04 \\
(1.70)^{* * *}\end{array}$ & $\begin{array}{c}1.05 \\
(2.85)^{*}\end{array}$ \\
\hline VTE & $\begin{array}{c}0.51 \\
(0.42)\end{array}$ & $\begin{array}{c}-0.35 \\
(-0.35)\end{array}$ & $\begin{array}{c}0.39 \\
(0.31)\end{array}$ & $\begin{array}{l}-0.31 \\
(-0.31)\end{array}$ & $\begin{array}{c}0.54 \\
(0.44)\end{array}$ & $\begin{array}{c}-0.28 \\
(-0.27)\end{array}$ & $\begin{array}{c}0.41 \\
(0.33)\end{array}$ & $\begin{array}{c}-0.26 \\
(-0.26)\end{array}$ \\
\hline OUV & $\begin{array}{c}6.67 \\
(2.68)^{*}\end{array}$ & $\begin{array}{c}0.79 \\
(0.66)\end{array}$ & $\begin{array}{c}6.05 \\
(2.29)^{*}\end{array}$ & $\begin{array}{c}0.50 \\
(0.39)\end{array}$ & $\begin{array}{c}6.19 \\
(2.86)^{*}\end{array}$ & $\begin{array}{c}1.44 \\
(1.22)^{* \star *}\end{array}$ & $\begin{array}{c}5.60 \\
(2.42)^{*}\end{array}$ & $\begin{array}{c}1.35 \\
(1.08)^{* * *}\end{array}$ \\
\hline LSDEXP & $\begin{array}{c}-1.60 \\
(-2.50)^{*}\end{array}$ & $\begin{array}{c}-1.34 \\
(-3.67)^{*}\end{array}$ & $\begin{array}{c}-1.70 \\
(-2.59)^{*}\end{array}$ & $\begin{array}{c}-1.39 \\
(-2.86)^{*}\end{array}$ & $\begin{array}{c}-1.58 \\
(-2.48)^{*}\end{array}$ & $\begin{array}{c}-1.21 \\
(-2.53)^{*}\end{array}$ & $\begin{array}{c}-1.68 \\
(-2.58)^{*}\end{array}$ & $\begin{array}{c}-1.22 \\
(-2.53)^{*}\end{array}$ \\
\hline LDETEXP & $\begin{array}{c}1.24 \\
(1.98)^{* *}\end{array}$ & $\begin{array}{c}1.09 \\
(2.33)^{*}\end{array}$ & $\begin{array}{c}3.00 \\
(1.16)^{* * *}\end{array}$ & $\begin{array}{c}2.43 \\
(1.06)^{* \star *}\end{array}$ & $\begin{array}{c}1.17 \\
(1.95)^{* *}\end{array}$ & $\begin{array}{c}1.03 \\
(2.19)^{*}\end{array}$ & $\begin{array}{c}2.99 \\
(1.15)^{* * *}\end{array}$ & $\begin{array}{c}1.50 \\
(0.66)\end{array}$ \\
\hline LDETEXP ${ }^{2}$ & & & $\begin{array}{c}-0.45 \\
(-0.70)\end{array}$ & $\begin{array}{c}-0.14 \\
(-0.60)\end{array}$ & & & $\begin{array}{c}-0.19 \\
(-0.72)\end{array}$ & $\begin{array}{c}-0.04 \\
(-0.21)\end{array}$ \\
\hline TINF & $\begin{array}{c}-0.04 \\
(-1.59)^{* * *}\end{array}$ & $\begin{array}{c}-0.06 \\
(-2.86)^{*}\end{array}$ & $\begin{array}{c}-0.04 \\
(-1.58)^{\star * *}\end{array}$ & $\begin{array}{c}-0.06 \\
(-2.83)^{*}\end{array}$ & $\begin{array}{c}-0.04 \\
(-1.59)^{* * *}\end{array}$ & $\begin{array}{c}-0.08 \\
(-3.40)^{*}\end{array}$ & $\begin{array}{c}-0.04 \\
(-1.58)^{* * \star}\end{array}$ & $\begin{array}{c}-0.08 \\
(-3.39)^{*}\end{array}$ \\
\hline Constante & $\begin{array}{l}-14.64 \\
(-1.81)^{\star \star}\end{array}$ & $\begin{array}{c}-9.46 \\
(-2.53)^{*}\end{array}$ & $\begin{array}{l}-18.89 \\
(-1.87)^{\star *}\end{array}$ & $\begin{array}{c}-12.7 \\
(-1.93)^{\star *}\end{array}$ & $\begin{array}{l}-14.17 \\
(-1.78)^{\star *}\end{array}$ & $\begin{array}{c}-3.91 \\
(-1.48)^{\star \star \star}\end{array}$ & $\begin{array}{l}-18.58 \\
(-1.85)^{\star *}\end{array}$ & $\begin{array}{c}-4.94 \\
(-0.89)\end{array}$ \\
\hline Nbre d'obs. & 230 & 230 & 230 & 230 & 230 & 230 & 230 & 230 \\
\hline $\mathrm{R}^{2}$ & 0.12 & 0.65 & 0.11 & 0.50 & 0.11 & 0.52 & 0.11 & 0.51 \\
\hline $\begin{array}{c}\text { F-stat } \\
\text { P-values }\end{array}$ & $\begin{array}{c}2.37 \\
(0.0111)\end{array}$ & & $\begin{array}{c}2.38 \\
(0.0109)\end{array}$ & & $\begin{array}{c}2.83 \\
(0.0026)\end{array}$ & & $\begin{array}{c}2.87 \\
(0.0022)\end{array}$ & \\
\hline $\begin{array}{c}\text { t-Haus } \\
\text { P-values }\end{array}$ & & $\begin{array}{c}23.03 \\
(0.0061)\end{array}$ & & $\begin{array}{c}30.02 \\
(0.0009)\end{array}$ & & $\begin{array}{c}35.87 \\
(0.0000)\end{array}$ & & $\begin{array}{c}17.62 \\
(0.0398)\end{array}$ \\
\hline
\end{tabular}

${ }^{*}$ significativité au seuil de $1 \%,{ }^{* *}$ significativité au seuil de $5 \%,{ }^{* *}$ significativité au seuil de $10 \%$, les valeurs entre (.) sont des t-students. 
Tableau 6 : Résultats des estimations du modèle linéaire et quadratique Indicateur d'endettement : dette extérieure en pourcentage du PIB Variable dépendante : taux de croissance du PIB réel par habitant

\begin{tabular}{|c|c|c|c|c|c|c|c|c|}
\hline \multirow{3}{*}{ Variables } & \multicolumn{4}{|c|}{ Avec Investissement } & \multicolumn{4}{|c|}{ Sans Investissement } \\
\hline & \multicolumn{2}{|c|}{ Linéaire } & \multicolumn{2}{|c|}{ quadratique } & \multicolumn{2}{|c|}{ Linéaire } & \multicolumn{2}{|c|}{ quadratique } \\
\hline & Within & GLS & Within & GLS & Within & GLS & Within & GLS \\
\hline Tcpibt-1 & $\begin{array}{c}-0.02 \\
(-0.41)\end{array}$ & $\begin{array}{l}0.005 \\
(0.11)\end{array}$ & $\begin{array}{l}-0.03 \\
(-0.70)\end{array}$ & $\begin{array}{c}-0.01 \\
(-0.20)\end{array}$ & $\begin{array}{c}-0.02 \\
(-0.44)\end{array}$ & $\begin{array}{c}0.03 \\
(0.76)\end{array}$ & $\begin{array}{l}-0.03 \\
(-0.70)\end{array}$ & $\begin{array}{c}0.03 \\
(0.61)\end{array}$ \\
\hline LTCD & $\begin{array}{c}-1.55 \\
(-1.19)^{* * *}\end{array}$ & $\begin{array}{c}-2.32 \\
(-2.67)^{*}\end{array}$ & $\begin{array}{l}-1.29 \\
(-0.99)\end{array}$ & $\begin{array}{c}-1.88 \\
(-2.08)^{*}\end{array}$ & $\begin{array}{c}-1.56 \\
(-1.22)^{* * *}\end{array}$ & $\begin{array}{c}-2.48 \\
(-2.85)^{*}\end{array}$ & $\begin{array}{l}-1.27 \\
(-0.99)\end{array}$ & $\begin{array}{c}-2.20 \\
(-2.43)^{*}\end{array}$ \\
\hline LTINV & $\begin{array}{c}0.05 \\
(2.24)^{*}\end{array}$ & $\begin{array}{c}2.20 \\
(2.04)^{*}\end{array}$ & $\begin{array}{c}0.16 \\
(0.12)\end{array}$ & $\begin{array}{c}2.60 \\
(2.36)^{*}\end{array}$ & & & & \\
\hline $\mathrm{LDCH}$ & $\begin{array}{c}2.48 \\
(1.20)^{* * * *}\end{array}$ & $\begin{array}{c}0.71 \\
(1.89)^{* *}\end{array}$ & $\begin{array}{c}2.53 \\
(1.22)^{*+*}\end{array}$ & $\begin{array}{c}0.82 \\
(2.16)^{*}\end{array}$ & $\begin{array}{c}2.45 \\
(1.35)^{* * *}\end{array}$ & $\begin{array}{c}0.91 \\
(2.48)\end{array}$ & $\begin{array}{c}2.64 \\
(1.46)^{* * *}\end{array}$ & $\begin{array}{c}1.01 \\
(2.68)^{*}\end{array}$ \\
\hline VTE & $\begin{array}{c}0.25 \\
(0.21)\end{array}$ & $\begin{array}{l}-0.85 \\
(-0.85)\end{array}$ & $\begin{array}{c}0.03 \\
(0.03)\end{array}$ & $\begin{array}{c}-0.86 \\
(-0.87)\end{array}$ & $\begin{array}{c}0.26 \\
(0.21)\end{array}$ & $\begin{array}{c}-0.78 \\
(-0.77)\end{array}$ & $\begin{array}{c}0.02 \\
(0.02)\end{array}$ & $\begin{array}{c}-0.78 \\
(-0.77)\end{array}$ \\
\hline OUV & $\begin{array}{c}5.31 \\
(2.23)^{*}\end{array}$ & $\begin{array}{l}0.15 \\
(0.12)\end{array}$ & $\begin{array}{c}4.62 \\
(1.91)^{* *}\end{array}$ & $\begin{array}{c}-0.25 \\
(-0.20)\end{array}$ & $\begin{array}{c}5.27 \\
(2.48)\end{array}$ & $\begin{array}{c}0.87 \\
(0.71)\end{array}$ & $\begin{array}{c}4.75 \\
(2.21)^{*}\end{array}$ & $\begin{array}{c}0.68 \\
(0.55)\end{array}$ \\
\hline LSDEXP & $\begin{array}{c}-1.10 \\
(-1.77)^{* * *}\end{array}$ & $\begin{array}{c}-0.96 \\
(-2.09)^{*}\end{array}$ & $\begin{array}{c}-1.25 \\
(-2.01)^{*}\end{array}$ & $\begin{array}{c}-1.09 \\
(-2.35)^{*}\end{array}$ & $\begin{array}{c}-1.09 \\
(-1.78)^{* *}\end{array}$ & $\begin{array}{c}-0.81 \\
(-1.78)^{* *}\end{array}$ & $\begin{array}{c}-1.26 \\
(-2.02)^{*}\end{array}$ & $\begin{array}{c}-0.88 \\
(-1.92)^{* *}\end{array}$ \\
\hline LDETPIB & $\begin{array}{c}0.57 \\
(0.89)\end{array}$ & $\begin{array}{c}0.64 \\
(1.31)^{*}\end{array}$ & $\begin{array}{c}4.08 \\
(1.75)^{* *}\end{array}$ & $\begin{array}{c}3.85 \\
(1.92)^{* *}\end{array}$ & $\begin{array}{c}0.57 \\
(0.90)\end{array}$ & $\begin{array}{c}0.54 \\
(1.12)^{* * * *}\end{array}$ & $\begin{array}{c}4.08 \\
(1.75)^{* *}\end{array}$ & $\begin{array}{c}2.74 \\
(1.39)^{*+* t}\end{array}$ \\
\hline LDETPIB $^{2}$ & & & $\begin{array}{c}-0.50 \\
(-1.56)^{* * *}\end{array}$ & $\begin{array}{c}-0.46 \\
(-1.65)\end{array}$ & & & $\begin{array}{c}-0.50 \\
(-1.56)^{* * *}\end{array}$ & $\begin{array}{c}-0.31 \\
(-1.15)^{* * *}\end{array}$ \\
\hline TINF & $\begin{array}{c}-0.04 \\
(-1.76)^{* *}\end{array}$ & $\begin{array}{c}-0.07 \\
(-2.86)^{*}\end{array}$ & $\begin{array}{c}-0.04 \\
(-1.57)^{* * * *}\end{array}$ & $\begin{array}{c}-0.06 \\
(-2.61)^{*}\end{array}$ & $\begin{array}{c}-0.04 \\
(-1.76)\end{array}$ & $\begin{array}{c}-0.08 \\
(-3.30)^{*}\end{array}$ & $\begin{array}{r}-0.04 \\
(-1.58)^{-* *}\end{array}$ & $\begin{array}{c}-0.07 \\
(-3.17)^{*}\end{array}$ \\
\hline Constante & $\begin{array}{c}-9.82 \\
(-1.23)^{* * * *}\end{array}$ & $\begin{array}{c}-6.63 \\
(-1.90)^{* *}\end{array}$ & $\begin{array}{l}-15.36 \\
(-1.77)^{* *}\end{array}$ & $\begin{array}{l}-13.02 \\
(-2.50)^{*}\end{array}$ & $\begin{array}{c}-9.78 \\
(-1.24)^{n * * *}\end{array}$ & $\begin{array}{l}-1.11 \\
(-0.50)\end{array}$ & $\begin{array}{l}-15.42 \\
(-1.78)^{* *}\end{array}$ & $\begin{array}{c}-4.82 \\
(-1.23)^{-* * *}\end{array}$ \\
\hline Nbre d'obs. & 230 & 230 & 230 & 230 & 230 & 230 & 230 & 230 \\
\hline $\mathbf{R}^{2}$ & 0.10 & 0.59 & 0.11 & 0.60 & 0.10 & 0.48 & 0.11 & 0.46 \\
\hline $\begin{array}{c}\text { F-stat } \\
\text { P-values }\end{array}$ & $\begin{array}{c}2.42 \\
(0.0096)\end{array}$ & & $\begin{array}{c}2.39 \\
(0.0106)\end{array}$ & & $\begin{array}{c}2.88 \\
(0.0022)\end{array}$ & & $\begin{array}{c}3.00 \\
(0.0015)\end{array}$ & \\
\hline $\begin{array}{l}\text { t-Haus } \\
\text { P-values }\end{array}$ & & $\begin{array}{c}27.22 \\
(0.0013)\end{array}$ & & $\begin{array}{c}30.87 \\
(0.0006)\end{array}$ & & $\begin{array}{c}42.88 \\
(0.0000)\end{array}$ & & $\begin{array}{c}129.06 \\
(0.0000)\end{array}$ \\
\hline
\end{tabular}

${ }^{*}$ significativité au seuil de $1 \%,{ }^{* *}$ significativité au seuil de $5 \%,{ }^{* * *}$ significativité au seuil de $10 \%$, les valeurs entre (.) sont des t-students. 


\section{Author' biography with Photo}

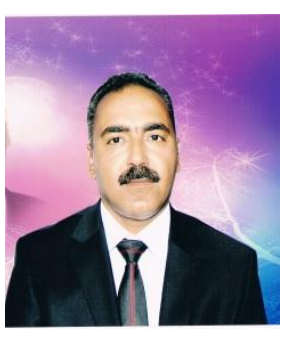

Tarek Ben Ali Assistant Professor in Economic Sciences Higher Institute of Business Administration

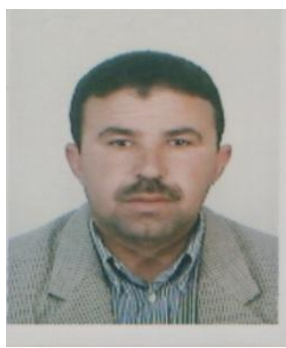

Ahmed Zidi Assistant Professor in Economic Sciences Higher Institute of Business Administration 